\title{
Functional neuroimaging of psychiatric disorders: exploring hidden behaviour*
}

From the outset, people have had high expectations of functional neuroimaging. Many will have been disappointed. After roughly a decade of widespread use, even an enthusiastic advocate must be diffident about the impact of the two most frequently used techniques - positron emission tomography (PET) and functional magnetic resonance imaging (fMRI) - upon clinical psychiatry. Perhaps this disappointment arises from an unrealistic expectation of what these techniques are able to tell us about the workings of the normal and the disordered brain. Anyone who hoped for intricate and unambiguous region-to-function mapping was always going to be disappointed. This expectation presupposes, among other things, a thorough understanding of the cognitive functions that are to be mapped onto the brain regions. This understanding, however, while developing, is still rudimentary. Mapping disorder along comparable lines is even more complex since it demands two levels of understanding. The first is of the healthy region-to-function mapping, the second of the disordered region-to-function mapping, which immediately demands a consideration of the nature of the function in the disordered state. After all, someone with schizophrenia, when confronted with a psychological task, might tackle it in a very different way, in terms of the cognitive strategies used, from a healthy person confronted with the same task. The observation that brain activity differs across the two individuals would only be interpretable insofar as one thoroughly understood the processes that each individual invoked in response to the task demands.

So, using functional neuroimaging to 'map' processes onto regions and interpreting group differences in these maps in terms of regional dysfunction is very difficult. Should we, therefore, be pessimistic about fMRI's potential contribution to clinical questions in psychiatry? I don't think that we should. In this issue of Psychological Medicine, two papers report fMRI studies illustrating ways in which the techniques may prove very useful indeed. In one, Blackwood and colleagues have explored the brain and behavioural responses to identifying the social and self-referential connotations of a series of statements (Blackwood et al. 2004). In the other, Werring and colleagues determined brain responses to visual stimulation in a group of people with visual loss of medically unexplained origin (Werring et al. 2004). As well as demonstrating the widening scope of functional neuroimaging applications to clinical questions in psychiatry, both studies highlight a potentially important application of the fMRI technique. This application lies in the provision of a sensitive outcome measure: one that may offer an index of occult or sub-behavioural cognitive processing anomalies in psychiatric disorders. The implications of this, as I will try to show, are exciting. These two, prima facie very different, studies actually use functional neuroimaging in ways that are, in one key respect, the same. In the first, Blackwood and colleagues have shown that, when behaviour is largely indistinguishable across groups of patients and controls, the imaging measurement is nevertheless sensitive to group differences. In the second, Werring and colleagues have shown that in a group of people who show subjective indices of visual problems, but in whom more objective clinical indices do not indicate pathology, fMRI may provide an objective measure of abnormal visual processing. In both cases, fMRI points to group differences that are not elicited using other behavioural measures. This is worth further examination. What are the implications of brain differences in the absence of behavioural differences? I will suggest that the capacity of functional

* Address for correspondence: Dr P. C. Fletcher, Wellcome Trust Senior Research Fellow in Clinical Science, University of Cambridge, Psychiatry Research Unit, Box 255, Level G2, Addenbrooke's Hospital, Cambridge CB2 2QQ, UK. (Email: pcf22@cam.ac.uk) 
neuroimaging to identify differences between groups or individuals, in the absence of other indices of difference, is a strength of the imaging techniques and will be important in the delineation and exploration of psychiatric illness. I will begin by considering the two studies in a little more detail.

Blackwood and colleagues report a factorial experimental manipulation in two groups of subjects: eight men with schizophrenia (and delusions present at the time of scanning) and eight agematched controls. During scanning, subjects were confronted either with neutral or with threatening statements. Some statements were potentially self-referential, others were more clearly non selfreferential. This generated four categories of statement: threatening/potentially self-referential (e.g. 'He is a liar'), threatening/non self-referential (e.g. 'Clive is a liar'), neutral/self-referential (e.g. 'He is resting') and neutral/non-self-referential (e.g. 'Clive is resting'). Subjects were required, in each case, to indicate whether they felt a statement was self-referential. There was little of the predicted behavioural difference between the two groups. What significant difference that there was arose from a single subject whose removal from the imaging analysis did not impact upon the findings. Thus, for various reasons, the patients and controls were scanned in the setting of behavioural performance that was largely indistinguishable. Yet brain activity patterns did distinguish the groups. In association with reading the potentially self-referential statements, deluded subjects showed significantly attenuated anterior cingulate cortex activation compared to control subjects. Posterior cingulate cortex, on the other hand, showed the reverse pattern: greater activation in the deluded subjects in association with self-referential statements. The authors interpret the failure of anterior cingulate cortex activation in terms of an impairment in self-reflection in the deluded state an interpretation that may be in keeping with previous imaging studies (e.g. Gallagher et al. 2000) suggesting the importance of the region in monitoring and mentalizing tasks.

In the second experiment, Werring and colleagues identified a group of five subjects in whom an objective evaluation of visual loss (visual-evoked potentials and pupillary light responses) was at odds with measurements based upon subjective claims (visual fields and acuity). All subjects fulfilled DSM-IV criteria for conversion disorder. This group and a group of seven volunteers with normal vision underwent fMRI either while viewing a rapidly flashing visual stimulus or while in darkness. The key differences between the two groups were that the subjects with medically unexplained visual deficits showed reduced levels of stimulus-induced activation in visual cortex and increased levels of activation in a number of cortical, striatal and thalamic areas. The authors interpret this pattern of activation as reflecting, in part, inhibitory modulation of primary visual areas, a finding that would be compatible with the small number of studies that have previously evaluated brain responses in people with medically unexplained symptoms (e.g. Costello et al. 1998; Spence et al. 2000; Hakala et al. 2002).

Both of these studies are considered by their authors to be preliminary. Each poses further questions about the implications of their findings. For example, Blackwood's observations invite us to ask whether the observations reflect the deluded state or the schizophrenia trait. Confronting this question would require observations in deluded patients with other diagnoses and in non-deluded patients with the same diagnosis. Ideally, as the authors suggest, the latter could be done in the same patients when the delusions had resolved. In Werring's paper, limitations lie in the small subject numbers and certain technical features that may be resolved in follow-up studies. One might also argue that, in future work, it would be useful additionally to compare medically unexplained visual loss with medically explained visual loss.

But we may leave aside problems in interpreting these imaging studies, and consider the fact that both point to the value of functional neuroimaging measures as a highly sensitive outcome variable. A compelling conclusion from both pieces of work is that fMRI sensitivity, in these contexts, exceeds concurrently or previously acquired subjective and objective measures in these people. Thus, Blackwood and colleagues used an online binary (yes or no) choice (does the statement refer to me?) and showed that this variable did not reliably differentiate the controls from the deluded participants. The fMRI data, however, did. Werring and colleagues have shown that while standard medical outcome measures do not differentiate these patients from the control subjects, the brain 
response to visual stimulation, expressed in regional fMRI signal responses, does. This is exciting. Recognizing a potential for functional neuroimaging to elucidate brain responses that are not reflected in other measures may have an impact upon the way in which the techniques contribute to psychiatric research and, ultimately, to clinical practice.

Of course, this may seem self-evident. After all, why would we want to use fMRI - an expensive, time-consuming and computationally demanding technique - simply to show that two conditions, two subjects or two groups differ when a simpler and cheaper behavioural measure (performance accuracy, reaction times, rating scales) might make this distinction equally well? One reason, of course, is that functional neuroimaging studies produce 'brain maps' and these can be very seductive. Brain-mapping experiments have constituted by far the most frequent use of PET and fMRI. They are characterized largely by experimental designs in which differing levels of regional brain activity are validated by corresponding changes in externally measurable behaviour. The goal of such experiments is to identify which bits of the brain 'do' the task and the observations are expressed in terms of evidence for regional specialization of function. For such an interpretation to be convincing, it might be argued, there must be independent evidence, from other behavioural measures, that the task design is truly manipulating the process or function of interest. There has been some, but not enough, debate about whether a brain activation that is observed in the absence of a behavioural validation, has any meaning (Wilkinson \& Halligan, 2004). As well as defending such an observation, I think that we should, in many circumstances, consider it desirable.

This approach - manipulate the process and see which brain regions wake up - used in conjunction with careful experimental psychology and neuropsychology, may certainly contribute to our understanding of functional specialization in the brain. However, it is not clear how easily it will translate when we move from functional imaging to dysfunctional imaging. In the latter, we are often trying to identify the site of pathology. But, particularly in the study of psychiatric illness, the functional neuroimaging tools are very limited in their capacity to make this identification with any real confidence (see Price \& Friston, 1999; Fletcher, 2000 for discussion). This is because any observable change in regional brain activity could feasibly be interpreted in three ways: it could be a cause of the disorder, a consequence of the disorder or a compensation for the disorder (Lewis, 2000). This ambiguity attends the most competently designed studies and it is very difficult to see how functional neuroimaging alone could resolve it. A functional neuroimaging study may certainly provide a signpost to the pathology but, standing alone, a fMRI observation that, say, an attenuation of language-related temporal lobe activation characterizes schizophrenia is no more proof that the temporal lobe houses a key causative abnormality in schizophrenia than would the observation of a slowed reaction time be indicative that schizophrenia is caused by slow reaction times. The observation of an abnormal activation would be the basis for a whole series of questions, most of them not directly answerable using functional neuroimaging. What is the nature of the language deficit, if any, that accompanies the temporal abnormality? Do non-schizophrenic people with identical deficits show identical temporal lobe activity? Do schizophrenic people with no measurable language deficit show the same pattern? What are the accompanying macro- and microstructural changes, if any, in this area of the temporal lobe?

This may seem a shocking admission - that fMRI has the capacity to say little, if anything, about causation in psychiatry. In fact, I believe that an acceptance of this limitation will contribute to a generally more realistic application of the techniques and that this, in turn, will guide experimentation most fruitfully. The message here is that the nice pictures produced by a functional neuroimaging experiment usually have the status of dependent variables and are limited accordingly. But, with respect to cognition, they are quite privileged dependent variables since they are multidimensional (having position as well as magnitude) and they are a lot closer to the action, cognitively speaking, than, say, performance or reaction-time measures. The latter are, after all, quite a long way downstream of cognitive processes.

With this in mind, I think that the two experiments in the current issue, and the admirable caution with which both sets of authors view their results is paradigmatic of the ways in which the particular attributes of the fMRI outcome variable may prove useful to psychiatry. Blackwood and colleagues 
have shown that, even when people with delusions appear to behave normally in a social judgement task, their brain indicates that they are processing the task differently. Of course, one could argue that, had the behavioural measurement been rendered more sensitive than a binary choice, then the external measures would have made this difference apparent. But imagine a study in which fMRI indicates differences that even a prolonged quest using other measures does not find. Such was the case in the study of Werring and colleagues (after all, the diagnosis of conversion disorder is dependent upon such a fruitless quest). The importance of this should not be overlooked. Conversion disorder is largely a diagnosis of exclusion: deriving from the absence of observable pathology. Ultimately, such a diagnosis has the same inconclusive status as any null finding. Moreover, since the diagnosis is expressed as an impairment in the absence of any observable physical deficit, it is one that many patients find distressing and unpalatable, believing it to carry connotations of psychological weakness or even pretence. Sadly this is a view that is not always confined to the patient. Perhaps the findings of Werring and colleagues, together with comparable findings may point to the possibility of fMRI providing measures that could ultimately render the diagnosis of conversion disorder a positive one, based upon observations made rather than observations not made.

Perhaps this may seem rather an unambitious way of using a highly sophisticated technique. After all, functional neuroimaging provides accurate spatial information and there have been many studies of psychiatric illness in which PET and fMRI have indicated apparent functional anomalies in key areas such as frontal cortex (e.g. Berman et al. 1988; Spence et al. 1998; Callicott et al. 2000), parietal cortex (e.g. Spence et al. 1997), anterior cingulate cortex (e.g. Dolan et al. 1995) and hippocampus (e.g. Heckers et al. 1998). More compelling still has been evidence from an elegant study in which the locus of functional abnormality in people with schizophrenia was related directly, within subjects, to structural deficits (Callicott et al. 2000). This points to the importance of adding complementary techniques to understand brain responses to differing contexts, in the quest for evidence of true regional pathology (e.g. Matsuo et al. 2002; Loo et al. 2003). However, I think that it is inescapable that, while we in psychiatry remain in states of cognitive, diagnostic and taxonomic disarray, a critical use of this highly sensitive variable is in trying to group or to prise apart clinical entities. Just as there are some elegant instances of the use of functional neuroimaging in testing competing cognitive models (Rees et al. 1997) so we should look to the techniques as a way of validating or testing competing groupings and diagnoses. Are delusions or hallucinations in mania functionally neuroanatomically distinct from those in schizophrenia or depression? Do patterns of brain activity support the current notions of descriptive sub-syndromes in schizophrenia (see Liddle et al. 1992; Honey et al. 2003)? Do they, indeed, support ideas that schizophrenia is a meaningful clinical entity? There are other exciting possibilities that attend our identification of changes in brain patterns with no immediate or easily measurable behavioural change. There is evidence, for example, that people genetically at risk of schizophrenia show changed patterns of brain activity in the face of apparently normal cognition (Callicott et al. 2003). This highlights the possibility of longitudinal studies identifying sub-behavioural characteristics of those at-risk subjects in whom symptoms may later occur. One recent exciting study shows that the technique may identify people in whom the first symptoms are beginning to emerge (Whalley et al. 2004). This is a potentially critical finding in view of the evidence that early treatment may be important. The prospect of fMRI contributing to individual-specific diagnostic and prognostic information, and thus having an impact upon treatment plans, is very appealing particularly in light of the fact that such information is currently so sketchy.

I have suggested that there is an important, but not always recognized, distinction between using functional neuroimaging to map the brain and using it to explore cognition. A comparable distinction exists between using it to identify the locus of psychiatric disorder and using it to explore the nature of the disordered processes. I claim no originality in making this distinction but argue that it is critical to bring it to the fore when considering the implications of psychiatric neuroimaging studies. In essence, the mapping enterprise, in seeking to identify the region of functional specialization, or the regional deficit underlying the illness, makes strong assumptions about the 
validity of the cognitive or the disease model. The imaging outcome is expressed in light of the model used and is interpreted accordingly. The alternative approach assumes that the cognitive/ disease model is the one under examination and the imaging outcome is not an end in itself (a 'map') but rather a measurement that informs our understanding of the model's validity. Using functional neuroimaging to test and refine our models of healthy and disordered cognitive function seems an important prelude to using it to map these models onto the brain.

P. C. FLETCHER

\section{ACKNOWLEDGEMENTS}

This work was supported by the Wellcome Trust. I am very grateful to Dr Garry Honey, with whom I have had interesting and useful discussions on the topics raised in this commentary.

\section{DECLARATION OF INTEREST}

None.

\section{REFERENCES}

Berman, K. F., Illowsky, B. P. \& Weinberger, D. R. (1988). Physiological dysfunction of dorsolateral prefrontal cortex in schizophrenia. IV. Further evidence for regional and behavioral specificity. Archives of General Psychiatry 45, 616-622.

Blackwood, N. J., Bentall, R. P., Ffytche, D. H., Simmons, A., Murray, R. M. \& Howard, R. J. (2004). Persecutory delusions and the determination of self-relevance: an fMRI investigation. Psychological Medicine 34, 591-596.

Callicott, J. H., Bertolino, A., Mattay, V. S., Langheim, F. J. P., Duyn, J., Coppola, R., Goldberg, T. E. \& Weinberger, D. R. (2000). Physiological dysfunction of the dorsolateral prefrontal cortex in schizophrenia revisited. Cerebral Cortex 10, 1078-1092.

Callicott, J. H., Egan, M. F., Mattay, V.S., Bertolino, A., Bone, A. D., Verchinksi, B. \& Weinberger, D. R. (2003). Abnormal fMRI response of the dorsolateral prefrontal cortex in cognitively intact siblings of patients with schizophrenia. American Journal of Psychiatry 160, 709-719.

Costello, A., Fletcher, P. C., Dolan, R. J., Frith, C. D. \& Shallice, T. (1998). The origins of forgetting in a case of isolated retrograde amnesia following a haemorrhage: evidence from functional imaging. Neurocase 4, 437-446.

Dolan, R. J., Fletcher, P., Frith, C. D., Friston, K. J., Frackowiak, R. S. \& Grasby, P. M. (1995). Dopaminergic modulation of impaired cognitive activation in the anterior cingulate cortex in schizophrenia. Nature 378, 180-182.

Fletcher, P. C. (2000). The functional neuroimaging of memory disorders. In Brain Mapping: The Disorders (ed. J. C. Mazziota, A. W. Toga and R. S. J. Frackowiak), pp. 201-215. Academic Press: San Diego.

Gallagher, H. L., Happe, F., Brunswick, N., Fletcher, P. C., Frith, U. \& Frith, C. D. (2000). Reading the mind in cartoons and stories: an fMRI study of 'theory of mind' in verbal and nonverbal tasks. Neuropsychologia 38, 11-21.

Hakala, M., Karlsson, H., Ruotsalainen, U., Koponen, S., Bergman, J., Stenman, H., Kelavuori, J.-P., Aalto, S., Kurki, T. \& Niemi, P. (2002). Severe somatisation in women is associated with altered cerebral glucose metabolism. Psychological Medicine 32, 1379-1385.

Heckers, S., Rauch, S. L., Goff, D., Savage, C. R., Schacter, D. L., Fischmann, A. \& Alpert, N. M. (1998). Impaired recruitment of the hippocampus during conscious recollection in schizoprehnia. Nature Neuroscience 1, 318-323.

Honey, G. D., Sharma, T., Suckling, J., Giampietro, V., Soni, W., Williams, S. C. R. \& Bullmore, E. T. (2003). The functional neuroanatomy of schizophrenic subsyndromes. Psychological Medicine 33, 1007-1018.
Lewis, D. A. (2000). Distributed disturbances in brain structure and function in schizophrenia. American Journal of Psychiatry 157, $1-2$.

Liddle, P. F., Friston, K. J., Frith, C. D., Hirsch, S. R., Jones, T. \& Frackowiak, R. S. (1992). Patterns of cerebral blood flow in schizophrenia. British Journal of Psychiatry 160, 179-186.

Loo, C. K., Sachdev, P. S., Haindl, W., Wen, W., Mitchell, P. B., Croker, V. M. \& Malhi, G. S. (2003). High (15 Hz) and low (1 Hz) frequency transcranial magnetic stimulation have different acute effect on regional cerebral blood flow in depressed patients. Psychological Medicine 33, 997-1006.

Matsuo, K., Kato, N. \& Kato, T. (2002). Decreased cerebral haemodynamic response to cognitive and physiological tasks in mood disorders as shown by near-infrared spectroscopy. Psychological Medicine 32, 1029-1037.

Price, C. J. \& Friston, K. J. (1999). Scanning patients with tasks they can perform. Human Brain Mapping 8, 102-108.

Rees, G., Frith, C. D. \& Lavie, N. (1997). Modulating irrelevant motion perception by varying attentional load in an unrelated task. Science 278, 1616-1619.

Spence, S. A., Brooks, D. J., Hirsch, S. R., Liddle, P. F., Meehan, J. \& Grasby, P. M. (1997). A PET study of voluntary movement in schizophrenic patients experiencing passivity phenomena (delusions of alien control). Brain 120, 1997-2011.

Spence, S. A., Crimlisk, H. L., Cope, H., Ron, M. A. \& Grasby, P. M. (2000). Discrete neurophysiological correlates in prefrontal cortex during hysterical and feigned disorder of movement [Letter]. Lancet 355, 1243-1244.

Spence, S. A., Hirsch, S. R., Brooks, D. J. \& Grasby, P. M. (1998). Prefrontal cortex activity in people with schizophrenia and control subjects. Evidence from positron emission tomography for remission of 'hypofrontality' with recovery from acute schizophrenia. British Journal of Psychiatry 172, 316-323.

Werring, D. J., Weston, L., Bullmore, E. T., Plant, G. T. \& Ron, M. A. (2004). Functional magnetic resonance imaging of the cerebral response to visual stimulation in medically unexplained visual loss. Psychological Medicine 34, 583-589.

Whalley, H. C., Simonotto, E., Flett, S., Marshall, I., Ebmeier, K. P., Owens, D. G. C., Goddard, N. H., Johnstone, E. C. \& Lawrie, S. M. (2004). fMRI correlates of state and trait effects in subjects at genetically enhanced risk of schizophrenia. Brain 127, 478-490.

Wilkinson, D. \& Halligan, P. (2004). The relevance of behavioural measures for functional imaging studies of cognition. Nature Reviews: Neuroscience 5, 67-73. 